VIDEOS IN CLINICAL MEDICINE

SUMMARY POINTS

Julie R. Ingelfinger, M.D., Editor

\title{
Administration of Supplemental Oxygen
}

\author{
Sundara Rengasamy, M.D., Bishoi Nassef, M.D., Federico Bilotta, M.D., \\ Francesco Pugliese, M.D., Ala Nozari, M.D., Ph.D., and Rafael Ortega, M.D.
}

The following text summarizes information provided in the video.

\section{OVERVIEW}

UPPLEMENTAL OXYGEN IS COMMONLY USED IN MODERN MEDICINE AND is fundamental to the treatment of hypoxemia. This print supplement and the related video focus on the administration of supplemental oxygen in adult patients in an acute care setting through a nasal cannula, simple face mask, face tent, nonrebreather face mask, Venturi mask, and high-flow nasal cannula (Table 1). Methods of oxygen administration that are not addressed in this video include noninvasive positive-pressure ventilation (e.g., continuous positive airway pressure and bilevel positive airway pressure), closed-system respiration through an endotracheal tube, and the administration of hyperbaric oxygen. Each technique may vary depending on institutional guidelines, the available equipment, and the manufacturer's instructions.

\section{INDICATIONS}

The most common indication for the administration of supplemental oxygen is acute or chronic hypoxemia. Causes include pulmonary infection, chronic obstructive pulmonary disease (COPD), congestive heart failure, pulmonary embolism, and shock. Oxygen therapy can also be beneficial in patients with burn injuries, carbon monoxide or cyanide poisoning, gas embolism, or other conditions. There are no absolute contraindications to the administration of supplemental oxygen.

\section{PHYSIOLOGY OF OXYGEN TRANSPORT}

Although a small fraction of oxygen is dissolved and transported in plasma, oxygen is largely transported in the blood, where it is bound to hemoglobin. The oxygen-hemoglobin dissociation curve shows the relationship between the partial pressure of arterial oxygen $\left(\mathrm{PaO}_{2}\right)$ and hemoglobin oxygen saturation. ${ }^{1}$

When the $\mathrm{PaO}_{2}$ is greater than approximately $60 \mathrm{~mm} \mathrm{Hg}$, the curve is relatively flat and the oxygen saturation does not change substantially in response to changes in the $\mathrm{PaO}_{2}$. However, as the $\mathrm{PaO}_{2}$ falls below $60 \mathrm{~mm} \mathrm{Hg}$, the slope of the curve becomes steeper, reflecting a sharp decrease in oxygen saturation that results in insufficient delivery of oxygen to the tissues. The administration of supplemental oxygen becomes critically important when the $\mathrm{PaO}_{2}$ approaches this threshold (Fig. 1).

Several physiologic factors can displace the oxyhemoglobin dissociation curve to the left or the right. ${ }^{2}$ Oxygen loading is favored when there is a leftward shift of the oxygen dissociation curve. The curve shifts to the left in the presence of hypothermia, decreased levels of 2,3-diphosphoglycerate (2,3-DPG), alkalosis, fetal hemoglobin, methemoglobinemia, and carbon monoxide poisoning. A rightward

From the Department of Anesthesiology, Boston Medical Center, Boston (S.R., B.N., A.N., R.O.), and the University of Rome, Rome (F.B., F.P.). Address reprint requests to Dr. Ortega at the Department of Anesthesiology, Boston Medical Center, 750 Albany St., Boston, MA 02118, or at rafael.ortega@bmc.org.

N Eng| J Med 2021;385:e9. DOI: 10.1056/NEJMvcm2035240

Copyright @ 2021 Massachusetts Medical Society. 


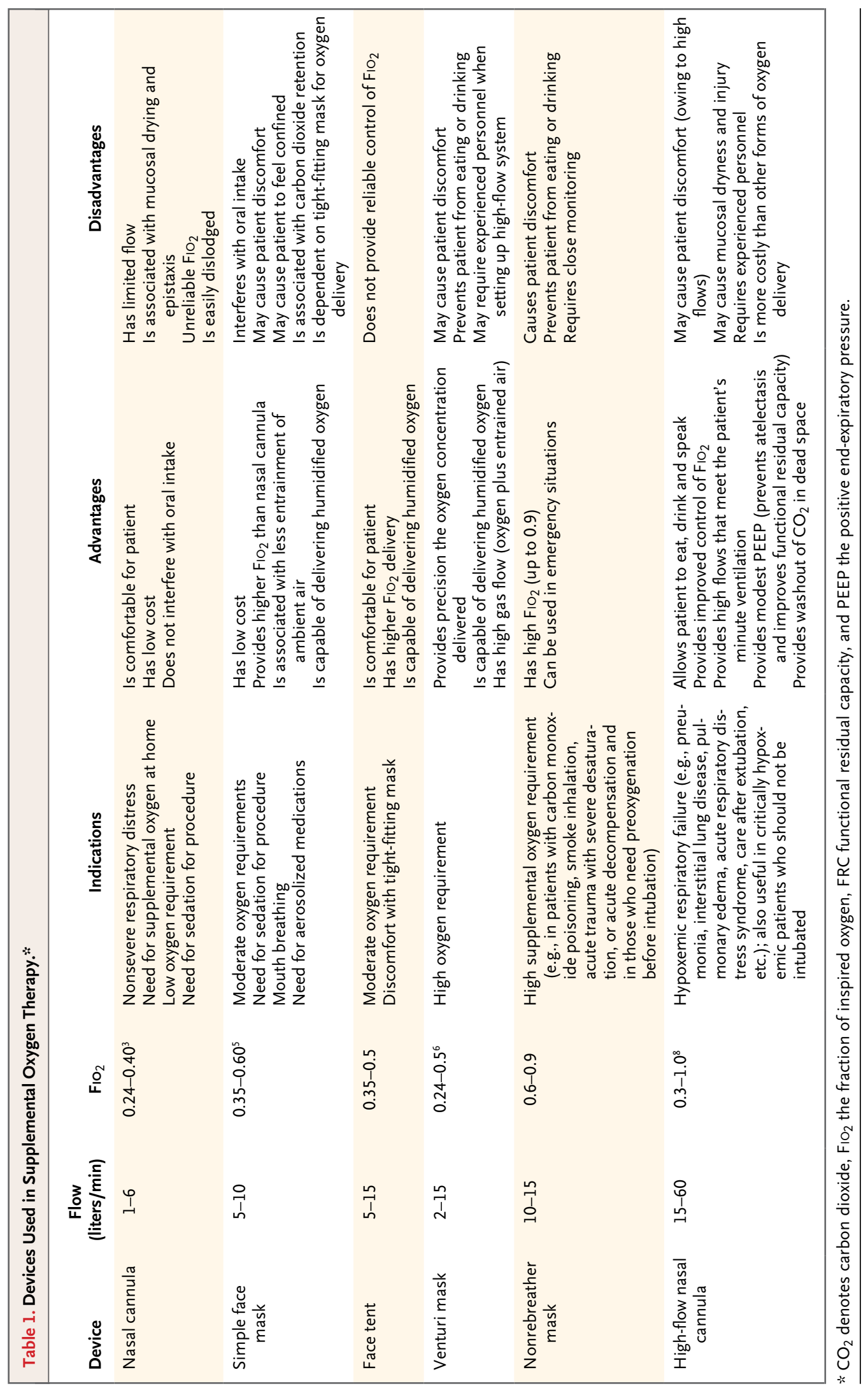

N ENGLJ MED 385;3 NEJM.ORG JULY 15, 2021

The New England Journal of Medicine 
shift, on the other hand, impairs oxygenation in the lungs and favors unloading of oxygen in the tissue. Rightward shift may be caused by hyperthermia, increased levels of 2,3-DPG, acidosis, or hypercapnia (i.e., the Bohr effect).

Pulse oximetry is particularly useful in monitoring the hemoglobin oxygen saturation. It is indicated in all clinical settings in which hypoxemia may occur.

\section{SOURCES OF SUPPLEMENTAL OXYGEN}

In many hospitals, a medical gas pipeline is the primary source of oxygen. When a pipeline is not available, E-cylinders are commonly used. A full E-cylinder with a pressure of 2000 pounds per square inch, or 14,000 kilopascals, contains approximately 660 liters of oxygen. Thus, at a set flow rate of 5 liters per minute, an E-cylinder would be able to deliver oxygen for 2 hours. H-cylinders are also commonly used in hospitals that do not have a central oxygen supply. When full, the $\mathrm{H}$-cylinders contain approximately 7000 liters of oxygen — approximately 10 times as much as that contained in an E-cylinder.

Some patients require long-term oxygen therapy and may benefit from having a portable oxygen concentrator in their home. ${ }^{3}$ This device entraps ambient air and compresses it, thereby delivering concentrated oxygen to the patient. An oxygen pulse is delivered to the user when inhalation is sensed as a negative fluctuation in pressure in the nasal cannula. Some oxygen concentrators are capable of delivering up to 10 liters of oxygen per minute.

Oxygen flow rate is typically measured with the use of a variable-orifice flow meter. The flow meter is attached to the oxygen source, and the measurement is obtained at the center of a floating ball. A fluted knob allows for adjustment of the flow. Flow meters have a tube that is tapered, with the smallest diameter at the bottom, thus creating a variable annular space between the ball and the inner wall of the tube. Since the flow depends on the viscosity and density of the gas, flow meters are constructed specifically for the delivery of a particular type of gas. Since the ball is suspended in the gas stream, these meters must be oriented vertically in order to function correctly.

E-cylinders are often equipped with click-style flow meters. The cylinders are easy to use, sturdy, and capable of operating at any angle because they are unaffected by gravity.

\section{APPROACHES TO THE ADMINISTRATION OF SUPPLEMENTAL OXYGEN}

\section{Nasal Cannula}

A nasal cannula is a flexible tube with two soft prongs that are inserted into the patient's nostrils. It is lightweight and can be used in the hospital, in the patient's home, or elsewhere. The tubing is usually looped behind the patient's ears and then brought in front of the neck. A sliding toggle is adjusted to secure the tubing in place. The main advantage of the nasal cannula is patient comfort, as it allows the patient to speak, drink, and eat easily with the cannula in place.

When oxygen is delivered through a nasal cannula, inspired ambient air mixes with the oxygen in varying proportions. In general, the fraction of inspired oxygen $\left(\mathrm{FIO}_{2}\right)$ that is above the normal atmospheric level increases by approximately $4 \%$ for every additional liter of oxygen administered per minute. However, an increase in minute ventilation - that is, the volume of gas inhaled or exhaled in 1 minute - or mouth breathing can dilute the oxygen and lower the $\mathrm{FiO}_{2}{ }^{4}$ Although oxygen can be administered through a nasal cannula at a flow rate of up to 6 liters per minute, lower flow rates are less likely to cause nasal dryness and discomfort.

Low-flow oxygen-delivery systems, such as nasal cannulas, do not provide very accurate estimates of the $\mathrm{FIO}_{2}$, particularly as compared with the delivery of oxygen

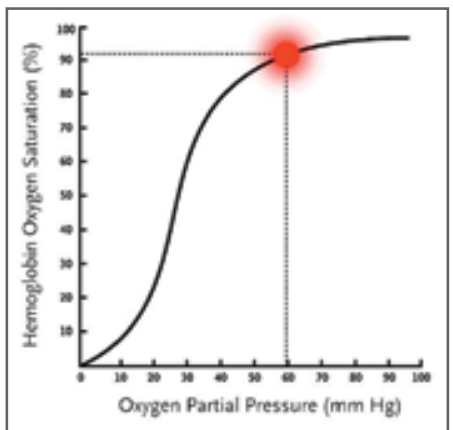

Figure 1. The Oxyhemoglobin Dissociation Curve.

The oxyhemoglobin dissociation curve represents the relationship between the partial pressure of arterial oxygen $\left(\mathrm{PaO}_{2}\right)$ and the oxygen saturation. The administration of supplemental oxygen becomes critically important when the $\mathrm{PaO}_{2}$ approaches $60 \mathrm{~mm} \mathrm{Hg}$. 
by means of a ventilator through an endotracheal tube. When the volume of inspired gas exceeds the oxygen flow (e.g., in patients with high minute ventilation), ambient air will make up the balance, thus decreasing the $\mathrm{FIO}_{2}$.

Oxygen can be administered through a nasal cannula at a flow rate of up to 6 liters per minute. Higher flow rates are more likely to cause nasal dryness, mucosal injury, and epistaxis.

\section{Simple Face Mask}

Like a nasal cannula, a simple face mask can be used to administer supplemental oxygen in a patient who is breathing spontaneously. The simple face mask does not have a reservoir. There are holes in both sides of the mask that allow the flow of ambient air during inspiration and the release of exhaled gas during expiration. The $\mathrm{FiO}_{2}$ is determined by the oxygen flow rate, the fit of the mask, and the patient's minute ventilation (Fig. 2).

In general, the administration of oxygen at a flow rate of 5 liters per minute generates an $\mathrm{FIO}_{2}$ of 0.35 to $0.60 . .^{5}$ Condensation of water vapor in the mask provides a visual cue of expiration and is rapidly cleared when an appropriate amount of fresh gas is provided. Disconnection of the oxygen tubing from the source or the use of a low oxygen flow rate can cause the patient to receive insufficient oxygen and to rebreathe exhaled carbon dioxide. These problems should be addressed immediately. Some patients may find that the face mask feels confining.

\section{Face Tent}

A face tent provides a controlled concentration of humidified oxygen through an open mask that fits beneath the patient's chin. The tent covers the nose and mouth without creating a seal around the nose. With a flow rate of 5 to 15 liters per minute, a face tent can provide an inspired oxygen concentration of up to $50 \%$. Its placement is ideal for patients who cannot wear a tight-fitting mask. However, the face tent's loose-fitting design and flow limitation can make it difficult to achieve precise control of the $\mathrm{FiO}_{2}$.

\section{Nonrebreather Face Mask}

A nonrebreather face mask is a face mask that has been modified to include an oxygen reservoir bag. A unidirectional valve allows oxygen to flow from the reservoir during inspiration but closes the reservoir during expiration, allowing it to fill with $100 \%$ oxygen from the source of fresh gas. A nonrebreather face mask can deliver an $\mathrm{FiO}_{2}$ between 0.6 and $0.9 .^{3}$

Nonrebreather face masks may be equipped with one or two side valves that close during inspiration to minimize the entrainment of ambient air. The valves open during expiration to prevent rebreathing of exhaled gas and reduce the risk of hypercarbia (Fig. 3).

The appropriate function of a nonrebreather mask that is equipped with two side valves is critically dependent on the flow of oxygen to the reservoir, as the unidirectional side valves prevent the inflow of ambient air through the vents. This arrangement is not recommended owing to the risk of suffocation if the fresh gas flow is insufficient or disrupted..

When oxygen is administered through a nonrebreather face mask, the flow should be set at 10 to 15 liters per minute and the patient should be monitored closely to ensure that there is an adequate flow of fresh gas from the reservoir. The reservoir bag should deflate only partially during inspiration. Complete deflation of the bag indicates insufficient gas flow and requires immediate attention. The delivery of supplemental oxygen through a face mask can be inefficient because of flow limitations.

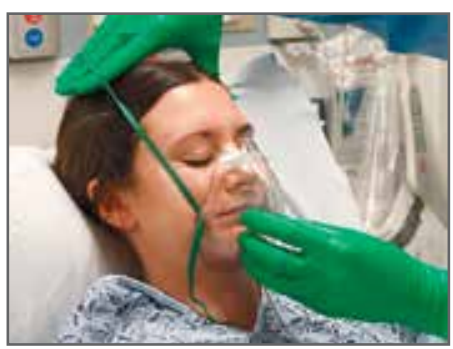

Figure 2. Simple Face Mask.

The simple face mask does not have a reservoir. The fraction of inspired oxygen is determined by the oxygen flow rate, the fit of the mask, and the patient's minute ventilation.

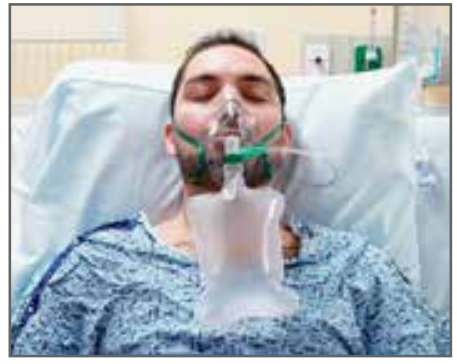

Figure 3. Nonrebreather and Partial Nonrebreather Face Masks.

Nonrebreather and partial nonrebreather face masks contain unidirectional valves that direct the flow of oxygen from the reservoir when the patient inhales and direct the exhaled gas to escape the mask to prevent the patient from rebreathing the gas. A nonrebreather face mask can deliver a fraction of inspired oxygen $\left(\mathrm{an} \mathrm{F}_{1} \mathrm{O}_{2}\right.$ ) between 0.6 and 0.9 . 


\section{Venturi Mask}

A Venturi mask takes advantage of the principle of jet mixing to entrain and blend ambient air with oxygen. As oxygen passes through a small orifice into a larger chamber, its velocity increases. This causes the ambient air to be entrained with the oxygen. Air entrainment can be controlled through manipulation of the orifice or the size of the air entrainment port, thereby producing a gas mixture with a predetermined $\mathrm{FIO}_{2}$.

The color-coded entrainment device at the base of the mask is designed to produce a fixed $\mathrm{FiO}_{2}$ of 0.24 to 0.50 provided that the required oxygen flow rate is set as specified by each manufacturer. ${ }^{6}$ After air entrainment, the overall rate of flow can be as high as 60 to 70 liters per minute. Since the color coding can vary across manufacturers, extra attention may be needed to set the correct flow of oxygen for each of the air-entrainment devices.

\section{High-Flow Nasal Cannula}

Like the face mask, the nasal cannula can be an inefficient means of oxygen delivery because of flow limitations, particularly in patients with a high inspiratory flow or high minute ventilation. A high-flow nasal cannula can deliver oxygen at a flow rate of more than 40 liters per minute. ${ }^{7}$ Although commercial high-flow nasal cannula systems are available in many designs, all require a flow generator, an active heated humidifier, and a single heated circuit. ${ }^{8}$ The oxygen is humidified and heated to a temperature of $37^{\circ} \mathrm{C}$ to prevent discomfort and mucosal injury.

Before a high-flow nasal cannula is placed on the patient, it should be assembled in accordance with the manufacturer's instructions (Fig. 4). There is a breathing circuit that consists of an air-oxygen blender, which is used to adjust the oxygen concentration, and a flow meter, which is used to adjust the flow. There is also a heated humidifier that continuously monitors the airway temperature and regulates the heating element. A sealed, heated wire within the circuit may be used to minimize condensation within the tubing. Sterile water is supplied from a prefilled disposable reservoir. Water flows from the reservoir into the humidification cartridge, where it is vaporized.

To administer oxygen through a high-flow nasal cannula, connect the oxygen tubing to the hospital oxygen pipeline, and connect the air tubing to the hospital air pipeline. Set the desired oxygen concentration on the air-oxygen blender and adjust the flow rate on the flow meter. Connect the high-flow nasal cannula to the breathing circuit. Ensure that there is adequate gas flow through the nasal prongs, and allow the gas to become warm and humidified before fitting the cannula to the patient. Position the nasal prongs in the nostrils, and secure the cannula. The prongs should not completely seal the nares; if they do, a smaller cannula should be used.

High-flow nasal cannulas generate a modest positive end-expiratory pressure. It has been suggested that their use may alleviate atelectasis and increase functional residual capacity. The efficacy of these devices may be enhanced by a washout effect, in which carbon dioxide is washed out from the anatomical dead space and replaced with oxygen-rich gas, thus reducing work of breathing and improving pulmonary mechanics. Moreover, the warmth and humidity provided by the cannula can improve patient comfort and facilitate airway clearance. The administration of supplemental oxygen through a high-flow nasal cannula can also reduce the need for endotracheal intubation and mechanical ventilation.

The use of high-flow nasal cannulas was initially avoided in patients with Covid-19, because the cannulas were thought to be associated with an increased risk of aerosolization and airborne transmission of the pathogen. However, emerging data suggest that this device can be used in patients with less severe forms of

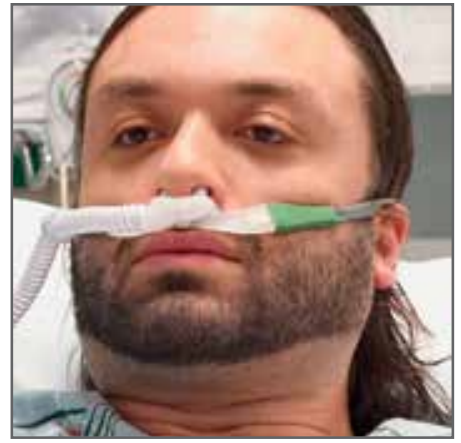

Figure 4. High-Flow Nasal Cannula.

High-flow nasal cannulas can deliver a fixed concentration of warm and humidified oxygen directly to the nasopharynx at a rate of more than 40 liters per minute. 
Covid-19 pneumonia without posing major risks to health care providers, as long as appropriate personal protective equipment is used and precautions against airborne transmission are taken ${ }^{9}$ (e.g., a surgical mask can be placed on the patient to reduce droplet deposition and the risk of particulate contamination).

\section{COMPLICATIONS}

The administration of oxygen is not without risk, and it is unclear which oxygenation targets are the most beneficial. Oxygen supports and accelerates combustion and causes materials to ignite and burn more avidly. During the administration of supplemental oxygen, it is important to avoid contact with sources of ignition and flammable materials. ${ }^{10}$

Increased oxygen tension can accelerate the production of reactive oxygen species, such as superoxide, hydroxyl radical, and hydrogen peroxide. Reactive oxygen species can impair the function of intracellular macromolecules and proteins, leading to cellular injury and tissue damage.

A high concentration of oxygen displaces nitrogen in the alveoli, and the oxygen in the alveoli may be absorbed, reducing the alveolar volume. Hyperoxia can result in absorption atelectasis, which can lead to increased physiological shunting and hypoxemia.

In some patients with COPD, oxygen therapy can cause retention of carbon dioxide. The precise mechanism through which this oxygen-induced hypercapnia occurs is poorly understood. It was originally thought to result from a reduction in the hypoxic stimulus of chemoreceptors. More recent data suggest that other factors, such as ventilation-perfusion mismatching or oxygen-induced changes in the carbon dioxide-hemoglobin dissociation curve (i.e., the Haldane effect) may be involved. ${ }^{11}$ If adequate oxygenation cannot be maintained in patients with such conditions without the development of life-threatening carbon dioxide retention, mechanical ventilation may be required.

\section{SUMMARY}

Supplemental oxygen is an important therapy for a broad range of clinical conditions. Oxygen can be administered through low-flow and high-flow devices that deliver a range of gas mixtures, with or without temperature and humidity control. It is important for clinicians to understand the functions and properties of the various devices used in oxygen delivery to ensure appropriate treatment and to avoid complications.

No potential conflict of interest relevant to this article was reported.

Disclosure forms provided by the authors are available with the full text of this article at NEJM.org.

\section{REFERENCES}

1. Severinghaus JW. Simple, accurate equations for human blood O2 dissociation computations. J Appl Physiol Respir Environ Exerc Physiol 1979;46:599-602.

2. Morgan TJ. The oxyhaemoglobin dissociation curve in critical illness. Crit Care Resusc 1999;1:93-100.

3. Hardavella G, Karampinis I, Frille A, Sreter K, Rousalova I. Oxygen devices and delivery systems. Breathe (Sheff) 2019;15(3):e108-e116.

4. Gibson RL, Comer PB, Beckham RW, McGraw CP. Actual tracheal oxygen concentrations with commonly used oxygen equipment. Anesthesiology 1976;44:71-3.

5. Wexler HR, Aberman A, Scott AA, Cooper JD. Measurement of intratracheal oxygen concentrations during face mask administration of oxygen: a modification for improved control. Can Anaesth Soc J 1975;22:417-31. 6. Speir WA Jr, Kaplan HM, Wier MA, Ellison LT. Oxygen concentration delivered by Venturi masks with in-line humidification. JAMA 1971;216:879-80.

7. Spoletini G, Alotaibi M, Blasi F, Hill NS. Heated humidified high-flow nasal oxygen in adults: mechanisms of action and clinical implications. Chest 2015;148:253-61.

8. Nishimura M. High-flow nasal cannula oxygen therapy devices. Respir Care 2019;64:735-42.

9. Li J, Fink JB, Ehrmann S. High-flow nasal cannula for COVID-19 patients: low risk of bio-aerosol dispersion. Eur Respir J 2020;55:2000892.

10. Angus DC. Oxygen therapy for the critically ill. N Engl J Med 2020;382:1054-6.

11. Abdo WF, Heunks LM. Oxygen-induced hypercapnia in COPD: myths and facts. Crit Care 2012;16:323.

Copyright (c) 2021 Massachusetts Medical Society. 